\title{
Interval Shannon Wavelet Collocation Method for Fractional Fokker-Planck Equation
}

\author{
Shu-Li Mei and De-Hai Zhu \\ College of Information and Electrical Engineering, China Agricultural University, Beijing 100083, China \\ Correspondence should be addressed to Shu-Li Mei; meishuli@163.com
}

Received 4 September 2013; Revised 5 October 2013; Accepted 6 October 2013

Academic Editor: Ming Li

Copyright (C) 2013 S.-L. Mei and D.-H. Zhu. This is an open access article distributed under the Creative Commons Attribution License, which permits unrestricted use, distribution, and reproduction in any medium, provided the original work is properly cited.

\begin{abstract}
Metzler et al. introduced a fractional Fokker-Planck equation (FFPE) describing a subdiffusive behavior of a particle under the combined influence of external nonlinear force field and a Boltzmann thermal heat bath. In this paper, we present an interval Shannon wavelet numerical method for the FFPE. In this method, a new concept named "dynamic interval wavelet" is proposed to solve the problem that the numerical solution of the fractional PDE is usually sensitive to boundary conditions. Comparing with the traditional wavelet defined in the interval, the Newton interpolator is employed instead of the Lagrange interpolation operator, so, the extrapolation points in the interval wavelet can be chosen dynamically to restrict the boundary effect without increase of the calculation amount. In order to avoid unlimited increasing of the extrapolation points, both the error tolerance and the condition number are taken as indicators for the dynamic choice of the extrapolation points. Then, combining with the finite difference technology, a new numerical method for the time fractional partial differential equation is constructed. A simple Fokker-Planck equation is taken as an example to illustrate the effectiveness by comparing with the Grunwald-Letnikov central difference approximation (GL-CDA).
\end{abstract}

\section{Introduction}

Due to the fact that $1 / f$ signal gains the increasing interests in the field of biomedical signal processing and engineering systems [1], the differential equations of fractional order appear more and more frequently in various research areas and engineering applications [2,3]. As a matter of fact, the applications of fractional differential equations and their corresponding time series have been developed in various fields of sciences and technologies $[4,5]$ in recent years, ranging from computer science to physics $[6,7]$. An effective and easy-to-use method for solving such equations is needed. However, known methods have certain disadvantages. Methods, described in detail in [3] for fractional differential equations of rational order, do not work in the case of arbitrary real order. On the other hand, there is an iteration method described in [8], which allows solution of fractional differential equations of arbitrary real order but it works effectively only for relatively simple equations, in addition to the series method. Up to now, most studies on the numerical methods for the fractional PDEs concentrate on the finite difference methods. Li [9] proposed an analytical method taking the fractal time series as the solution to a differential equation of fractional order or a response of a fractional system or a fractional filter driven with a white noise in the domain of stochastic processes and gave the exact solution of impulse response to a class of fractional oscillators [10]. According to this idea, Li and his coresearchers solved many problems in science and technology [11-14]. In addition, Wavelet numerical method is another way to get the solution of the fractional PDEs. In fact, the wavelet transform theory has been widely used in numerical analysis such as PDEs-based image processing [1517], option pricing model [18], integrodifferential operators [19-23], and other nonlinear PDEs [24-28]. The wavelet functions possess many excellent numerical properties, such as orthogonality, interpolation, smoothness, and compact support, which are helpful in improving numerical accuracy and efficiency. In recent decades, many wavelets which have compact support, smoothness, and other properties have 
been constructed. Among these wavelets, Shannon wavelet is paid little attention in applications as it does not possess compact support property although it possesses orthogonality, smooth continuity, and analytical expression. Cattani studied the properties of the Shannon wavelet function, which possesses many advantages such as orthogonality, continuous and differentiable. It also has the advantage over the Hermite DAF in that it is an interpolating function, producing matrix equations that have the potential to be relatively sparse. In addition, the second order approximation of a $C^{2}$-function, based on Shannon wavelet functions, is given [29]. The approximation is compared with the wavelet reconstruction formula and the error of approximation is explicitly computed [30].

A perceived disadvantage of the Shannon scaling function is that it tends to zero quite slowly as $|x| \rightarrow \infty$. A direct consequence of this is that when calculating the derivatives, a large number of the nodal values will contribute significantly. It is for this reason that Hoffman et al. [31] have suggested using the Shannon-Gabor wavelet, which introduces the Gaussian window function to improve the compact support property of Shannon wavelet function in required precision range. However, the presence of the Gaussian window destroys the orthogonal properties possessed by the Shannon wavelet, effectively worsening the approximation to a Dirac delta function.

Comparing with the common PDEs, the solutions of the fractional PDEs are more sensitive to the boundary condition. Using the wavelet transform defined in infinite domain to solve the engineering problems in finite interval, the wavelet transform coefficients at the boundary are usually very large. It will bring server boundary effect which affects the calculation accuracy and efficiency. Vasilyev and Paolucci [32] construct an interval wavelet using external wavelets, which can decrease the boundary effect to some extent. Based on the same principle, a more general construction method for the interval interpolation wavelet $[33,34]$ was given in the framework of generalized variational principle and has been widely used in many areas [35-37]. But the choice of parameter $L$ (that is the amount of the external collocation points) was not discussed in detail. It just points out that the value of $L$ should be taken between 1 and 3 based on experience. In fact, the value of $L$ depends on the smoothness and derivative of the approximated function at boundary points. That is, if the approximated function is the solution of the diffusion PDEs with respect to the time parameter, the value of $L$ should be taken dynamically. In addition, we should take into account that the impact of the external collocation points to the condition number of the system of the discretized algebraic equations. So, it is necessary to construct a dynamic interval wavelet in solving the PDEs with dynamic boundary conditions such as the fractional PDEs.

In this paper, a dynamic interval Shannon wavelet collocation method for the fractional FPDs is proposed. In this method, the relation between the parameter $L$ and the wavelet approximation error was discussed based on the interpolation error theory, and an adaptive choice procedure on $L$ was constructed. Therefore, the so-called dynamic interval Shannon wavelet is constructed. Next, based on the Grünwald-Letnikov definition of the fractional order derivative, we construct a Shannon wavelet numerical method for the fraction Fokker-Planck equation.

\section{Fractional Fokker-Planck Equation}

The fractional Fokker-Planck equation has been used in many physical transport problems which take place under the influence of an external force field $[2,38]$.

In the presence of an external force field $F(x)=-v^{\prime}(x)$, the evolution of a test particle is usually described in terms of the Fokker-Planck equation (FPE)

$$
\begin{array}{r}
\frac{\partial u(x, t)}{\partial t}={ }_{0} D_{t}^{1-\alpha}\left[\frac{\partial}{\partial x} \frac{\nu^{\prime}(x)}{m \eta_{\alpha}}+K_{\alpha} \frac{\partial^{2}}{\partial x^{2}}\right] u(x, t), \\
a \leq x \leq b, 0 \leq t \leq T,
\end{array}
$$

which defines the probability $u(x, t)$ of finding the particle at a certain position $x$ at a given time $t$. $m$ denotes the mass of the diffusing particle, $K_{\alpha}>0$ denotes the generalized diffusion coefficient with dimension $\left[K_{\alpha}\right]=\mathrm{cm}^{2} \mathrm{sec}^{-\alpha}$, and $\eta_{\alpha}$ is the generalized friction coefficient with dimension $\left[\eta_{\alpha}\right]=$ $\sec ^{\alpha-2}$. The corresponding initial condition is

$$
u(x, 0)=\varphi(x), \quad a \leq x \leq b,
$$

and the boundary conditions are

$$
u(a, t)=p_{1}(t), \quad u(b, t)=p_{2}(t), \quad 0<t \leq T .
$$

Equation (1) uses the Riemann-Liouville fractional derivative of order $1-\alpha$, defined by

$$
\begin{gathered}
{ }_{0} D_{t}^{1-\alpha} u(x, t)=\frac{1}{\Gamma(\alpha)} \frac{\partial}{\partial t} \int_{0}^{1} \frac{u(x, \eta)}{(t-\eta)^{1-\alpha}} d \eta \\
0 \leq \alpha<1
\end{gathered}
$$

where $\Gamma(\alpha)$ is the gamma function.

According to the properties of the Riemann-Liouville fractional derivative, it is easy to know that, if $(x, t) \in$ $C_{x, t}^{2,1}([a, b] \times[0, T]),(1)$ can be rewritten as follows:

$$
\begin{gathered}
D_{t}^{\alpha} u(x, t)-\frac{u(x, 0) t^{-\alpha}}{\Gamma(1-\alpha)}=\left[\frac{\partial}{\partial x} \frac{\nu^{\prime}(x)}{m \eta_{\alpha}}+K_{\alpha} \frac{\partial^{2}}{\partial x^{2}}\right] u(x, t), \\
a \leq x \leq b, 0 \leq t \leq T
\end{gathered}
$$

Metzler et al. [2] proposed three implicit approximations for solving (5) as follows. 
(1) The Grünwald-Letnikov expansion and the backward Euler implicit approximation (GL-BDIA)

$$
\begin{gathered}
\tau^{-\alpha}\left[u_{i}^{n}+\sum_{k=1}^{n-1} g_{k} u_{i}^{n-k}-\sum_{k=0}^{n-1} g_{k} u_{i}^{0}\right] \\
=\frac{f_{i} u_{i}^{n}-f_{i-1} u_{i-1}^{n}}{h}+K_{\alpha} \frac{u_{i+1}^{n}-2 u_{i}^{n}+u_{i-1}^{n}}{h^{2}}, \\
i=1,2, \ldots, M-1 \\
u_{0}^{n}=p_{1}\left(t_{n}\right), \quad u_{M}^{n}=p_{2}\left(t_{n}\right), \quad n \geq 1, \\
g_{k}=\left(1-\frac{1+\alpha}{k}\right) g_{k-1}, \quad g_{0}=1, \\
f_{i}=f\left(x_{i}\right)=\frac{v^{\prime}\left(x_{i}\right)}{m \eta_{\alpha}},
\end{gathered}
$$

where $h=(b-a) / M, \tau=T / N, x_{i}=a+i h$, and $t_{n}=n \tau . M$ and $N$ are positive integers. The local truncation error is $O(\tau+h)$.

(2) $L_{1}$-approximation and the central difference implicit approximation $\left(L_{1}\right.$-CDIA)

$$
\begin{gathered}
\frac{\tau^{-\alpha}}{\Gamma(2-\alpha)}\left[u_{i}^{n}+\sum_{k=1}^{n-1}\left(a_{n-k-1}-a_{n-k}\right) u_{i}^{k}-a_{n-1} u_{i}^{0}\right] \\
=\frac{f_{i+1} u_{i+1}^{n}-f_{i-1} u_{i-1}^{n}}{2 h}+K_{\alpha} \frac{u_{i+1}^{n}-2 u_{i}^{n}+u_{i-1}^{n}}{h^{2}} \\
i=1,2, \ldots, M-1 \\
u_{i}^{0}=\varphi\left(x_{i}\right), \quad 1 \leq i \leq M, \\
u_{0}^{n}=p_{1}\left(t_{n}\right), \quad u_{M}^{n}=p_{2}\left(t_{n}\right), \quad n \geq 1 \\
a_{k}=(k+1)^{1-\alpha}-k^{1-\alpha}, \\
f_{i}=f\left(x_{i}\right)=\frac{v^{\prime}\left(x_{i}\right)}{m \eta_{\alpha}} .
\end{gathered}
$$

The local truncation error is $O\left(\tau^{2-\alpha}+h^{2}\right)$.

(3) $L_{1}$-approximation and the backward difference implicit approximation $\left(L_{1}\right.$-BDIA $)$

$$
\begin{gathered}
\frac{\tau^{-\alpha}}{\Gamma(2-\alpha)}\left[u_{i}^{n}+\sum_{k=1}^{n-1}\left(a_{n-k-1}-a_{n-k}\right) u_{i}^{k}-a_{n-1} u_{i}^{0}\right] \\
=\frac{f_{i} u_{i}^{n}-f_{i-1} u_{i-1}^{n}}{h}+K_{\alpha} \frac{u_{i+1}^{n}-2 u_{i}^{n}+u_{i-1}^{n}}{h^{2}} \\
i=1,2, \ldots, M-1 \\
u_{i}^{0}=\varphi\left(x_{i}\right), \quad 1 \leq i \leq M \\
u_{0}^{n}=p_{1}\left(t_{n}\right), \quad u_{M}^{n}=p_{2}\left(t_{n}\right), \quad n \geq 1
\end{gathered}
$$

$$
\begin{gathered}
f_{i}=f\left(x_{i}\right)=\frac{v^{\prime}\left(x_{i}\right)}{m \eta_{\alpha}}, \\
a_{k}=(k+1)^{1-\alpha}-k^{1-\alpha} .
\end{gathered}
$$

The local truncation error is $O\left(\tau^{2-\alpha}+h\right)$.

In fact, (6)-(2) are not perfect approximation as the boundary effect is not taken into account. So, it will introduce boundary effect in solving the PDEs with the Nuemann boundary conditions. It is well known that the finite difference method is equivalent to the Faber-Schauder wavelet collocation method, so the construction method of the dynamic interval wavelet introduced in this paper can also be used to deal with the boundary problem in the finite difference method.

According to the Shannon sample theory, it can improve the calculation precision that combining the GrünwaldLetnikov expansion or $L_{1}$-approximation of the fractional derivative in (5) with the Shannon scaling function as the weight function instead of the various difference operators as follows:

$$
\begin{gathered}
\frac{\tau^{-\alpha}}{\Gamma(2-\alpha)}\left[u_{i}^{n}+\sum_{k=1}^{n-1}\left(a_{n-k-1}-a_{n-k}\right) u_{i}^{k}-a_{n-1} u_{i}^{0}\right] \\
=\sum_{i=0}^{2^{J}}\left[f_{i} u_{i} w^{\prime}(x)+K_{\alpha} u_{i} w^{\prime \prime}(x)\right]
\end{gathered}
$$

$i=1,2, \ldots, 2^{J}$, consider $J$ is the positive integer.

$$
u_{i}^{0}=\varphi\left(x_{i}\right), \quad 1 \leq i \leq M
$$

$$
\begin{gathered}
u_{0}^{n}=p_{1}\left(t_{n}\right), \quad u_{M}^{n}=p_{2}\left(t_{n}\right), \quad n \geq 1 \\
f_{i}=f\left(x_{i}\right)=\frac{v^{\prime}\left(x_{i}\right)}{m \eta_{\alpha}} \\
a_{k}=(k+1)^{1-\alpha}-k^{1-\alpha} .
\end{gathered}
$$

\section{Construction of the Interval Interpolation Wavelet}

3.1. Shannon Wavelet and Shannon-Gabor Wavelet. The representation of Shannon wavelet is based upon approximating the Dirac delta function as a band-limited function and is given by

$$
\phi(x)=\frac{\sin (\pi x)}{\pi x}
$$

and the Shannon-Gabor scaling function is defined as [17]

$$
G(x)=\frac{\sin (\pi x)}{\pi x} \exp \left(-\frac{x^{2}}{2 \sigma^{2}}\right), \quad \sigma>0,
$$

where $\sigma$ is the window size.

Consider a one-dimensional function $f(x), x \in[a, b]$. A discrete point sequence of the variable $x$ is defined as 


$$
x_{n}=a+\frac{b-a}{2^{j}} \cdot n, \quad j \in \mathbf{Z}, n=0,1,2, \ldots, 2^{j},
$$

and the corresponding discrete point sequence of the scaling function $\phi(x)$ and $G(x)$ can be defined, respectively, as

$$
\begin{aligned}
\phi_{j, n}(x)=\phi_{j}\left(x-x_{n}\right)= & \frac{\sin \left(2^{j} \pi /(b-a)\right)\left(x-x_{n}\right)}{\left(2^{j} \pi /(b-a)\right)\left(x-x_{n}\right)}, \\
G_{j, n}(x)=G_{j}\left(x-x_{n}\right)= & \frac{\sin \left(2^{j} \pi /(b-a)\right)\left(x-x_{n}\right)}{\left(2^{j} \pi /(b-a)\right)\left(x-x_{n}\right)} \\
& \times \exp \left(-\frac{2^{2^{j}-1}\left(x-x_{n}\right)^{2}}{r^{2}(b-a)^{2}}\right),
\end{aligned}
$$

where $r=2^{j} \sigma /(b-a)$.
The first and second order derivatives of $\phi_{j}\left(x-x_{n}\right)$ at the discrete point $x_{k}$ are

$$
\begin{gathered}
\phi_{j}^{\prime}\left(x_{k}-x_{n}\right)= \begin{cases}0, & k=n, \\
\frac{2^{j} \cos [\pi(k-n)]}{(k-n)(b-a)}, & k \neq n,\end{cases} \\
\phi_{j}^{\prime \prime}\left(x_{k}-x_{n}\right)= \begin{cases}-\frac{\pi^{2}}{3\left((b-a) / 2^{j}\right)^{2}}, & k=n, \\
-\frac{2 \cos [\pi(k-n)]}{\left((b-a) / 2^{j}\right)^{2}(k-n)^{2}}, & k \neq n .\end{cases}
\end{gathered}
$$

And the first and second order derivatives of $G_{j}\left(x-x_{n}\right)$ at the discrete point $x_{k}$ are

$$
\begin{gathered}
G_{j}^{\prime}\left(x_{k}-x_{n}\right)= \begin{cases}0, & k=n \\
\frac{2^{j} \cos [\pi(k-n)] \exp \left[-(k-n)^{2} / 2 r^{2}\right]}{(k-n)(b-a)}, & k \neq n\end{cases} \\
G_{j}^{\prime \prime}\left(x_{k}-x_{n}\right)= \begin{cases}-\frac{3+\pi^{2} r^{2}}{3 r^{2}\left((b-a) / 2^{j}\right)^{2}}, & k=n \\
-\frac{2 \cos [\pi(k-n)] \exp \left[-(k-n)^{2} / 2 r^{2}\right]}{\left((b-a) / 2^{j}\right)^{2}}\left[\frac{1}{(k-n)^{2}}+\frac{1}{r^{2}}\right], & k \neq n .\end{cases}
\end{gathered}
$$

In fact, there is no difference between the construction method of the Interval Shannon wavelet and the interval Shannon-Gabor wavelet. So, we just take one uniform symbol $w(x)$ as the representation of the Shannon wavelet and the Shannon-Gabor wavelet in the following.
3.2. Interval Interpolation Wavelet. According to the definition of the interval wavelet, the interval interpolation basis functions can be expressed as:

$$
w_{j k}(x)= \begin{cases}\phi\left(2^{j} x-k\right)+\sum_{n=-L+1}^{-1} a_{n k} \phi\left(2^{j} x-n\right), & k=0, \ldots, L \\ \phi\left(2^{j} x-k\right), & k=L+1, \ldots, 2^{j}-L-1 \\ \phi\left(2^{j} x-k\right)+\sum_{n=2^{j}+1}^{2^{j}+L-1} b_{n k} \phi\left(2^{j} x-n\right), & k=2^{j}-L, \ldots, 2^{j},\end{cases}
$$

where,

$$
\begin{gathered}
a_{n k}=\prod_{i=L-1, i \neq k}^{-1} \frac{x_{j, n}-x_{j, i}}{x_{j, k}-x_{j, i}}, \quad b_{n k}=\prod_{i=2^{j}+1, i \neq k}^{2^{j}+1+L} \frac{x_{j, n}-x_{j, i}}{x_{j, k}-x_{j, i}} \\
x_{j, k}=k \frac{x_{\max }-x_{\min }}{2^{j}}, \quad k \in \mathbb{Z},
\end{gathered}
$$

where $L$ is the amount of the external collocation points, the amount of discrete points in the definition domain is $2^{j}+$ $1(j \in \mathbb{Z})$, and $\left[x_{\min }, x_{\max }\right]$ is the definition domain of the approximated function.
Equations (16) and (17) show that the interval wavelet is derived from the domain extension. The supplementary discrete points in the extended domain are called external points. The value of the approximated function at the external points can be obtained by Lagrange extrapolation method. Using the interval wavelet to approximate a function, the boundary effect can be left in the supplementary domain; that is, the boundary effect is eliminated in the definition domain.

According to (16) and (17), the interval wavelet approximant of the function $f(x) x \in\left[x_{\min }, x_{\max }\right]$ can be expressed as 


$$
\begin{aligned}
f_{j}(x) & =\sum f_{j}\left(x_{n}\right) w_{j}\left(2^{j} x-n\right), \\
x_{n} & =x_{\min }+n \frac{x_{\max }-x_{\min }}{2^{j}} .
\end{aligned}
$$

$f_{j}\left(x_{n}\right)$ is the given value at the discrete point $x_{n}$. At the external points, $f_{j}\left(x_{n}\right)$ can be obtained by extrapolation; that is

$$
f_{j}\left(x_{n}\right)= \begin{cases}\sum_{k=0}^{L-1}\left(f_{j}\left(x_{k}\right) \prod_{i=0, i \neq k}^{L-1} \frac{x_{n}-x_{i}}{x_{k}-x_{i}}\right), & n=-1, \ldots,-L \\ \sum_{k=2^{j}-L+1}^{2^{j}}\left(f_{j}\left(x_{k}\right) \prod_{i=2^{j}-L+1, k \neq i}^{2^{j}} \frac{x_{n}-x_{i}}{x_{k}-x_{i}}\right), & n=2^{j}+1, \ldots, 2^{j}+L .\end{cases}
$$

So the interval wavelet approximant of $f(x)$ can be rewritten as

$$
\begin{aligned}
f_{j}(x)= & \sum_{n=-L}^{-1}\left(\sum_{k=0}^{L-1} f_{j}\left(x_{k}\right) \prod_{i=0}^{L-1} \frac{x_{n}-x_{i}}{x_{k}-x_{i}}\right) \omega\left(2^{j} x-n\right) \\
& +\sum_{n=0}^{2^{j}} f_{j}\left(x_{k}\right) \omega\left(2^{j} x-n\right) \\
& +\sum_{n=2^{j}+1}^{2^{j}+L}\left(\sum_{k=2^{j}-L}^{2^{j}} f_{j}\left(x_{k}\right) \prod_{i=2^{j}-L}^{2^{j}} \frac{x_{n}-x_{i}}{x_{k}-x_{i}}\right) \omega\left(2^{j} x-n\right) .
\end{aligned}
$$

Let

$$
\begin{aligned}
& L S_{L}\left(x_{n}\right)=\sum_{k=0}^{L-1} f_{j}\left(x_{k}\right) \prod_{i=0}^{L-1} \frac{x_{n}-x_{i}}{x_{k}-x_{i}} \\
& L E_{L}\left(x_{n}\right)=\sum_{k=2^{j}-L}^{2^{j}} f_{j}\left(x_{k}\right) \prod_{i=2^{j}-L}^{2^{j}} \frac{x_{n}-x_{i}}{x_{k}-x_{i}},
\end{aligned}
$$

then

$$
\begin{aligned}
f_{j}(x)= & \sum_{n=-L}^{-1} L S_{L}\left(x_{n}\right) \omega\left(2^{j} x-n\right) \\
& +\sum_{n=0}^{2^{j}} f_{j}\left(x_{k}\right) \omega\left(2^{j} x-n\right) \\
& +\sum_{n=2^{j}+1}^{2^{j}+L} L E_{L}\left(x_{n}\right) \omega\left(2^{j} x-n\right) .
\end{aligned}
$$

$L S_{L}\left(x_{n}\right)$ and $L E_{L}\left(x_{n}\right)$ correspond to the left and the right external points, respectively. They are obtained by Lagrange extrapolation using the internal collocation points near the boundary. So, the interval wavelet's influence on the boundary effect can be attributed to Lagrange extrapolation. It should be pointed out that we did not care about the reliability of the extrapolation. The only function of the extrapolation is enlarging the definition domain of the given function which can avoid the boundary effect occurred in the domain. Therefore, we can discuss the choice of $L$ by means of Lagrange inner-and extrapolation error polynomial as follows:

$$
\begin{array}{r}
R_{L}(x)=\frac{f^{(L+1)}(\xi)}{(L+1) !} \prod_{i=0}^{L}\left(x-x_{i}\right), \text { for some } \xi \text { between } \\
x, x_{0}, \ldots, x_{L} .
\end{array}
$$

Equation (23) indicates that the approximation error is both related to the smoothness and the gradient of the original function near the boundary. Setting different $L$ can satisfy the error tolerance.

3.3. Adaptive Interval Interpolation Wavelet. The interval interpolation wavelet is often used to solve the diffusion PDEs with Neumann boundary conditions. The smoothness and gradient of the PDE's solution usually vary with the time parameter. If the parameter $L$ is a constant, we have to take a bigger value in order to obtain results with higher calculation precision. But the bigger $L$ usually introduces the famous Gibbs phenomena into the numerical solution, which usually results in the algorithm becoming invalid. In addition, the bigger $L$ will bring much more calculation. To keep higher numerical precision and save calculation, the best way is to design a procedure that $L$ can vary with the curve's smoothness and gradient dynamically.

In this dynamic procedure, the error estimation equation (23) can be taken as the criterion about $L$. But in most cases, we cannot know the smoothness and the derivative's order of the original function. This can be solved by substituting the difference coefficient for the derivative. This is coincident with the Newton interpolation equation which is equivalent with Lagrange interpolation equation. In addition, the Lagrange interpolation algorithm has no inheritance which is the key feature of Newton interpolation. So, the basis function has to be calculated repeatedly as interpolation points are added into the calculation, which increases the computation complexity greatly. In contracst to the Lagrange method, the advantage of Newton interpolation method is that the Newton divided difference form is employed, which can produce a mathematically equivalent result by using recurrence relations, which reduces the number of compute operation, especially the multiplication. So it is convenient using the Newton interpolation method to construct the dynamic procedure. 
3.3.1. Newton Interpolation. The expression of Newton interpolation can be written as

$$
\begin{aligned}
N_{n}(x)= & f\left(x_{0}\right)+\left(x-x_{0}\right) f\left(x_{0}, x_{1}\right) \\
& +\left(x-x_{0}\right)\left(x-x_{1}\right) f\left(x_{0}, x_{1}, x_{2}\right)+\cdots \\
& +\left(x-x_{0}\right)\left(x-x_{1}\right) \cdots\left(x-x_{n-1}\right) \\
& \times f\left(x_{0}, x_{1}, \ldots, x_{n}\right) .
\end{aligned}
$$

Substituting the Newton interpolation instead of the Lagrange interpolation into (22) can be rewritten as

$$
\begin{aligned}
f_{j}(x)= & \sum_{n=-L}^{-1}\left(N S_{L}\left(x_{n}\right)\right) \omega\left(2^{j} x-n\right) \\
& +\sum_{n=0}^{2^{j}} f_{j}\left(x_{n}\right) \omega\left(2^{j} x-n\right) \\
& +\sum_{n=2^{j}+1}^{2^{j}+L}\left(N E_{L}\left(x_{n}\right)\right) \omega\left(2^{j} x-n\right),
\end{aligned}
$$

where

$$
\begin{aligned}
N S_{L}\left(x_{n}\right)= & f\left(x_{0}\right)+\left(x_{n}-x_{0}\right) f\left(x_{0}, x_{1}\right) \\
& +\left(x_{n}-x_{0}\right)\left(x_{n}-x_{1}\right) f\left(x_{0}, x_{1}, x_{2}\right)+\cdots \\
& +\left(x_{n}-x_{0}\right)\left(x_{n}-x_{1}\right) \cdots\left(x_{n}-x_{L-1}\right) \\
& \times f\left(x_{0}, x_{1}, \ldots, x_{L}\right), \\
N S_{R}\left(x_{n}\right)= & f\left(x_{2^{j}}\right)+\left(x_{n}-x_{2^{j}}\right) f\left(x_{2^{j}}, x_{2^{j}-1}\right) \\
& +\left(x_{n}-x_{2^{j}}\right)\left(x_{n}-x_{2^{j}-1}\right) \\
& \times f\left(x_{2^{j}}, x_{2^{j}-1}, x_{2^{j}-2}\right)+\cdots \\
& +\left(x_{n}-x_{2^{j}}\right)\left(x_{n}-x_{2^{j}-1}\right) \cdots\left(x_{n}-x_{2^{j}-L}\right) \\
& \times f\left(x_{2^{j}}, x_{2^{j}-1}, \ldots, x_{2^{j}-L}\right) .
\end{aligned}
$$

3.3.2. Relation between the Newton Interpolation Error and the Choice of $L$. It is well known that the Newton interpolation is equivalent to the Lagrange interpolation. The corresponding error estimation can be expressed as

$$
R_{n}(x)=\left(x-x_{0}\right)\left(x-x_{1}\right) \cdots\left(x-x_{n}\right) f\left(x, x_{0}, \ldots, x_{n}\right) .
$$

And the simplest criterion to terminate the dynamic choice on $L$ is $\left|R_{n}(x)\right| \leq T_{a}$ ( $T_{a}$ is the absolute error tolerance). Obviously, it is difficult to define $T_{a}$ which should meet with the precision requirement of all approximated curves. In fact, the difference coefficient $f\left(x, x_{0}, \ldots, x_{n}\right)$ can be used directly as the criterion; that is

$$
\left|f\left(x, x_{0}, \ldots, x_{n}\right)\right|<\varepsilon .
$$

As mentioned above, once the curves with lower order smoothness are approximated by higher order polynomial expression, the errors will become bigger on the contrary. In fact, even if the $L$ is infinite, the computational precision cannot be satisfied except by increasing computational complexity. To avoid this, we design the termination procedure of dynamic choice about $L$ as follows:

$$
\begin{aligned}
& \text { If } f\left(x_{0}, x_{1}\right)<T_{a} \text {, then } L=1 \\
& \text { else if } f\left(x_{0}, x_{1}, x_{2}\right)<T_{a} \text { or } f\left(x_{0}, x_{1}, x_{2}\right)< \\
& f\left(x_{0}, x_{1}\right) \text {, then } L=2 \\
& \text { else if } f\left(x_{0}, x_{1}, x_{2}, x_{3}\right)<T_{a} \text { or } f\left(x_{0}, x_{1}, x_{2}, x_{3}\right)< \\
& f\left(x_{0}, x_{1}, x_{2}\right) \text {, then } L=3
\end{aligned}
$$

3.3.3. L and the Condition Number of the System of Algebraic Equations. In the field of numerical analysis, the condition number of a function with respect to an argument measures how much the output value of the function can change for a small change in the input argument. This is used to measure how sensitive a function is to changes or errors in the input and how much error in the output results from an error in the input. It is no doubt that the choice of $L$ can change the condition number of the system of algebraic equations discretized by the wavelet interpolation operator or the finite difference method. Therefore, the choice of $L$ should take the condition number into account. In fact, if the condition number $\operatorname{cond}(A)=10^{k}$, then you may lose up to $k$ digits of accuracy on top of what would be lost to the numerical method due to loss of precision from arithmetic methods [24]. According to the general rule of thumb, the choice should follow the rule as follows:

$$
\frac{\operatorname{Cond}\left(A_{L+1}\right)}{\operatorname{Cond}\left(A_{L}\right)}<10 \text {. }
$$

3.3.4. Relation between L and Computation Complexity. The computational complexity of interpolation calculation is not proportional to the increasing points. The former is mainly up to the computation amount of $\left(x-x_{0}\right)\left(x-x_{1}\right) \cdots\left(x-x_{n}\right)$ and the derivative operations. Obviously, according to (5), the increase in computational complexity is $O\left(L^{3}\right)$ when the number of extension points $L$ increases by 1 . But the computational complexity of adaptively increasing collocation points is related to the different wavelet functions. For the wavelet with compact support property such as Daubechies wavelet and Shannon wavelet, the value of $L$ is impossible to be infinite. For Haar wavelet which has no smoothness property, $L$ can be taken as 0 at most since it need not to be extended. For Faber-Schauder wavelet, $L$ can be taken as 1 at most. For Daubechies wavelet, $L$ can be taken as different values according to the order of vanishing moments, but it must be finite. For the wavelets without compact support property, $L$ can take value dynamically, such as Shannon wavelet. The computational complexity of increasing points is mainly up to the wavelet function of itself. 


\section{Numerical Results and Discussion}

Fractional Fokker-Planck equation is a typical fractional PDE, which is often used to describe a subdiffusive behavior of a particle under the combined influence of external nonlinear force field, and a Boltzmann thermal heat bath. This section considers the accuracy and efficiency of the proposed method for a fractional Fokker-Planck equation. Comparisons are made with results obtained with Chen's finite difference approximations and the exact analytic solution.

It has been pointed out that the finite difference approximation formats proposed in [2] are not perfect as they do not take the boundary problems into account. In this section, we take the Grünwald-Letnikov expansion and the central difference implicit approximation (GL-CDIA) to solve the example. That is,

$$
\begin{aligned}
& \tau^{-\alpha}\left[u_{i}^{n}+\sum_{k=1}^{n-1} g_{k} u_{i}^{n-k}-\sum_{k=0}^{n-1} g_{k} u_{i}^{0}\right] \\
& =\frac{f_{i+1} u_{i+1}^{n}-f_{i-1} u_{i-1}^{n}}{2 h}+K_{\alpha} \frac{u_{i+1}^{n}-2 u_{i}^{n}+u_{i-1}^{n}}{h^{2}}, \\
& i=1,2, \ldots, M-1 \text {, } \\
& \tau^{-\alpha}\left[u_{0}^{n}+\sum_{k=1}^{n-1} g_{k} u_{0}^{n-k}-\sum_{k=0}^{n-1} g_{k} u_{0}^{0}\right] \\
& =\frac{4 f_{1} u_{1}^{n}-3 f_{0} u_{0}^{n}-f_{2} u_{2}^{n}}{2 h}+K_{\alpha} \frac{u_{0}^{n}-2 u_{1}^{n}+u_{2}^{n}}{h^{2}} \text {, } \\
& \tau^{-\alpha}\left[u_{M}^{n}+\sum_{k=1}^{n-1} g_{k} u_{M}^{n-k}-\sum_{k=0}^{n-1} g_{k} u_{M}^{0}\right] \\
& =\frac{f_{M-2} u_{M-2}^{n}-4 f_{M-1} u_{M-1}^{n}+3 f_{M} u_{M}^{n}}{2 h} \\
& +K_{\alpha} \frac{u_{M-2}^{n}-2 u_{M-1}^{n}+u_{M}^{n}}{h^{2}} \\
& u_{i}^{0}=\varphi\left(x_{i}\right), \quad 1 \leq i \leq M, \\
& u_{0}^{n}=p_{1}\left(t_{n}\right), \quad u_{M}^{n}=p_{2}\left(t_{n}\right), \quad n \geq 1, \\
& g_{k}=\left(1-\frac{1+\alpha}{k}\right) g_{k-1}, \quad g_{0}=1 \\
& f_{i}=f\left(x_{i}\right)=\frac{v^{\prime}\left(x_{i}\right)}{m \eta_{\alpha}} .
\end{aligned}
$$

According to the wavelet collocation method, the fractional Fokker-Planck equation can be approximately represented as

$$
\begin{gathered}
\tau^{-\alpha}\left[u_{j}\left(x_{i}, t_{n}\right)+\sum_{k=1}^{n-1} g_{k} u_{j}\left(x_{i}, t_{n-k}\right)\right. \\
\left.-\sum_{k=0}^{n-1} g_{k} u_{j}\left(x_{i}, t_{0}\right)\right]
\end{gathered}
$$

$$
\begin{aligned}
&=f^{\prime}\left(x_{i}\right) u_{j}\left(x_{i}, t_{n}\right) \\
&+\sum_{m=0}^{2^{j}} u_{j}\left(x_{m}, t_{n}\right) \\
& \quad \times\left[w^{\prime}\left(x_{i}-x_{m}\right)+K_{\alpha} w^{\prime \prime}\left(x_{i}-x_{m}\right)\right],
\end{aligned}
$$

where $i=0,1,2, \ldots 2^{j}$. Let

$$
\begin{aligned}
& V_{j}^{n}=\left(u_{j}\left(x_{0}, t_{n}\right), u_{j}\left(x_{1}, t_{n}\right), \ldots, u_{j}\left(x_{2^{j}}, t_{n}\right)\right)^{T} \text {, } \\
& F=\operatorname{diag}\left(f^{\prime}\left(x_{0}\right), f^{\prime}\left(x_{1}\right), \ldots, f^{\prime}\left(x_{2^{j}}\right)\right), \\
& W_{1} \\
& =\left[\begin{array}{cccc}
w^{\prime}\left(x_{0}-x_{0}\right) & w^{\prime}\left(x_{0}-x_{1}\right) & \cdots & w^{\prime}\left(x_{0}-x_{2^{j}}\right) \\
w^{\prime}\left(x_{1}-x_{0}\right) & w^{\prime}\left(x_{1}-x_{1}\right) & \cdots & w^{\prime}\left(x_{1}-x_{2^{j}}\right) \\
\vdots & \vdots & \ddots & \vdots \\
w^{\prime}\left(x_{2^{j}}-x_{0}\right) & w^{\prime}\left(x_{2^{j}}-x_{1}\right) & \cdots & w^{\prime}\left(x_{2^{j}}-x_{2^{j}}\right)
\end{array}\right], \\
& =\left[\begin{array}{cccc}
w^{\prime \prime}\left(x_{0}-x_{0}\right) & w^{\prime \prime}\left(x_{0}-x_{1}\right) & \cdots & w^{\prime \prime}\left(x_{0}-x_{2^{j}}\right) \\
w^{\prime \prime}\left(x_{1}-x_{0}\right) & w^{\prime \prime}\left(x_{1}-x_{1}\right) & \cdots & w^{\prime \prime}\left(x_{1}-x_{2^{j}}\right) \\
\vdots & \vdots & \ddots & \vdots \\
w^{\prime \prime}\left(x_{2^{j}}-x_{0}\right) & w^{\prime \prime}\left(x_{2^{j}}-x_{1}\right) & \cdots & w^{\prime \prime}\left(x_{2^{j}}-x_{2^{j}}\right)
\end{array}\right]
\end{aligned}
$$

Then the system of (31) can be expressed as the matrix format:

$$
\begin{aligned}
\left(W_{1}\right. & \left.+K_{\alpha} W_{2}+F-\tau^{-\alpha} I\right) V_{j}^{n} \\
& =\sum_{k=1}^{n-1} g_{k} V_{j}^{n-k}-\sum_{k=0}^{n-1} g_{k} V_{j}^{0} .
\end{aligned}
$$

Next, we will discuss the precision of the method proposed in this paper with numerical experience. Consider the FokkerPlanck equation as follows:

$$
\begin{gathered}
\frac{\partial u(x, t)}{\partial t}={ }_{0} \mathbf{D}_{t}^{1-\alpha}\left[\frac{\partial}{\partial x}(-1)+\frac{\partial^{2}}{\partial x^{2}}\right] u(x, t), \\
0 \leq x \leq 1, t>0,
\end{gathered}
$$

with the initial condition

$$
u(x, 0)=x(1-x), \quad 0 \leq x \leq 1
$$

and the boundary conditions

$$
\begin{array}{ll}
u(0, t)=-\frac{3 t^{\alpha}}{\Gamma(1+\alpha)}-\frac{2 t^{2 \alpha}}{\Gamma(1+2 \alpha)}, & t>0, \\
u(1, t)=-\frac{t^{\alpha}}{\Gamma(1+\alpha)}-\frac{2 t^{2 \alpha}}{\Gamma(1+2 \alpha)}, & t>0 .
\end{array}
$$



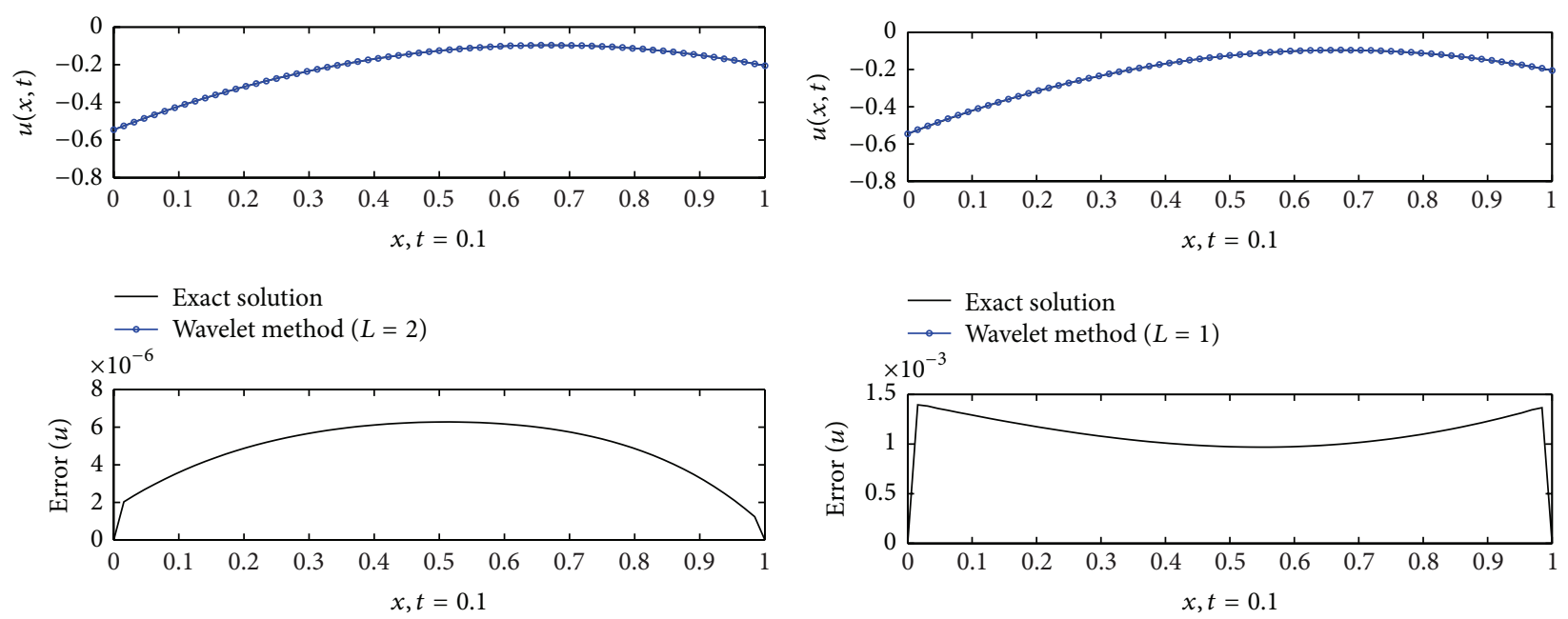

(a) $j=6, L=2$, condition number is $76.7074, e_{1}=6.2776 \times 10^{-6}$, and $e_{2}=4.9985 \times 10^{-6}$

(b) $j=6, L=1$, condition number is $31.2950, e_{1}=1.4 \times 10^{-3}$, and $e_{2}=1.1 \times 10^{-3}$

FIGURE 1: Wavelet collocation method with constant $L(\alpha=0.8)$.
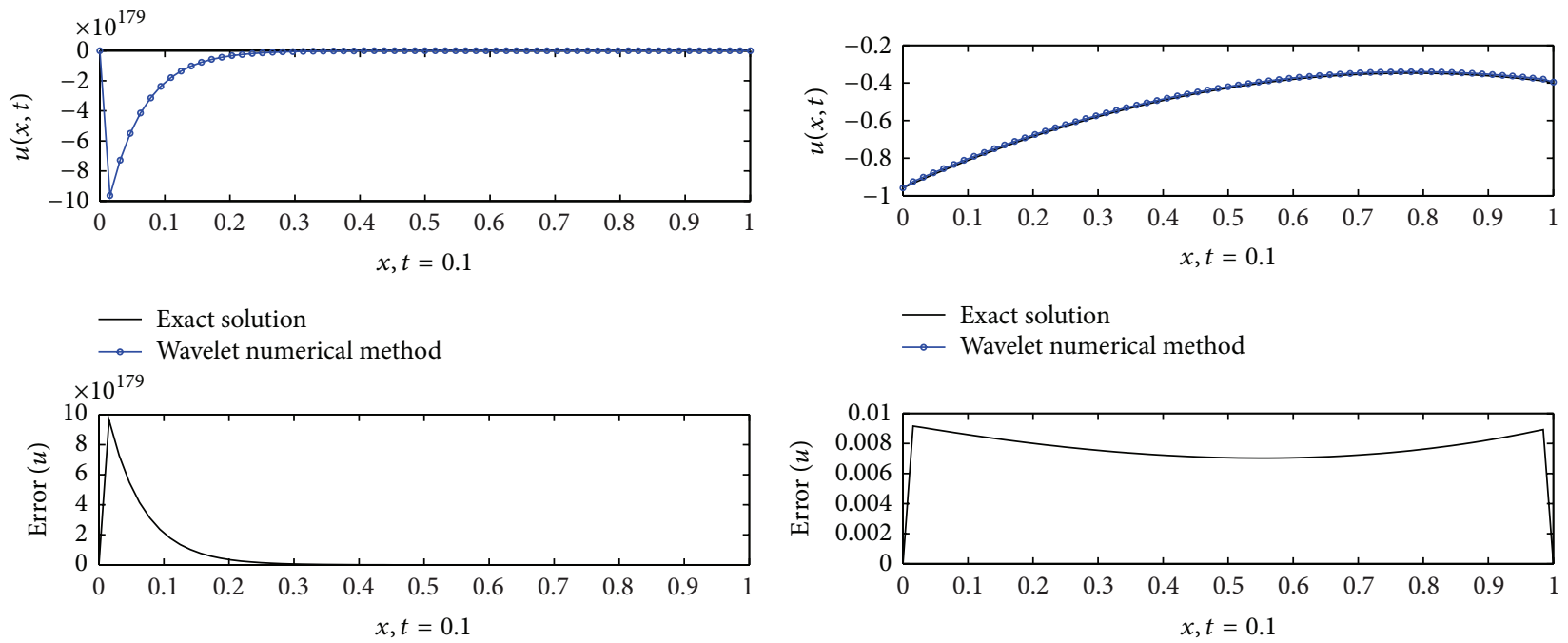

(a) $j=6, L=2$, and $\tau=0.0001$

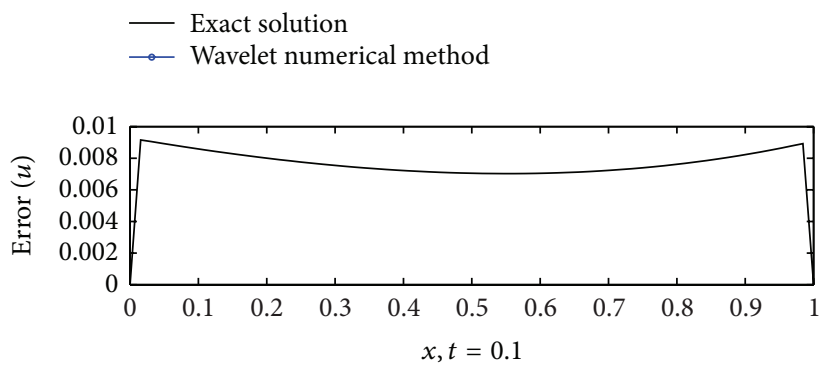

(b) $j=6, L=1$, and $\tau=0.0001$

FIGURE 2: Wavelet collocation method with constant $L(\alpha=0.6)$.

The exact analytic solution is

$$
u(x, t)=x(1-x)+(2 x-3) \frac{t^{\alpha}}{\Gamma(1+\alpha)}-\frac{2 t^{2 \alpha}}{\Gamma(1+2 \alpha)}
$$

All the comparisons in this section are made qualitatively by comparing the calculation precision in the same time step and space mesh grid size. The first measure of error $e_{1}$ is given by

$$
e_{1}=\left\|V_{j}^{n}-V_{\text {exact }}^{n}\right\|_{\infty}
$$

which provides a measure of the accuracy of the solution near the boundary. The second measure of error $e_{2}$ is given by

$$
e_{2}=\sqrt{\frac{1}{2^{j}+1} \sum_{i=0}^{2^{j}}\left(u\left(x_{i}\right)-u_{\text {exact }}\left(x_{i}\right)\right)^{2}}
$$

which provides a general measure of the accuracy of the solution over the main body of the distribution and was often used to investigate the accuracy of the FEM.

The comparisons between the static interval ShannonGabor wavelets with $L=1$ and $L=2$ are showen in Figure 1. The boundary effect of the interval wavelet with $L=2$ (Figure $1(\mathrm{a})$ ) is almost eliminated compared 


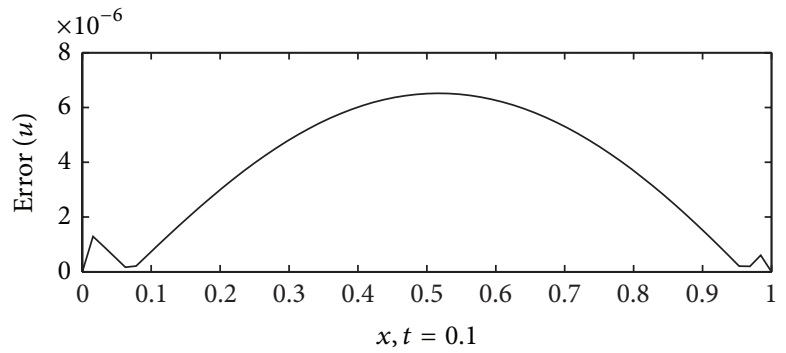

(a) Adapt interval wavelet collocation method

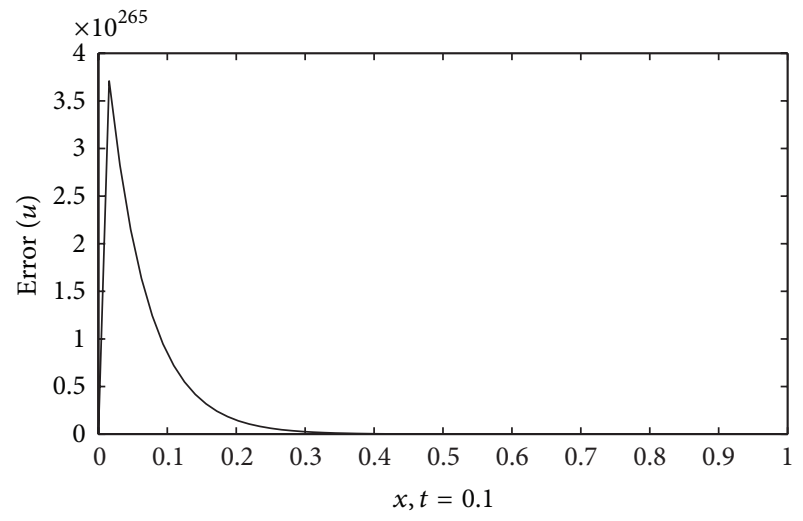

(b) Interval-finite difference method

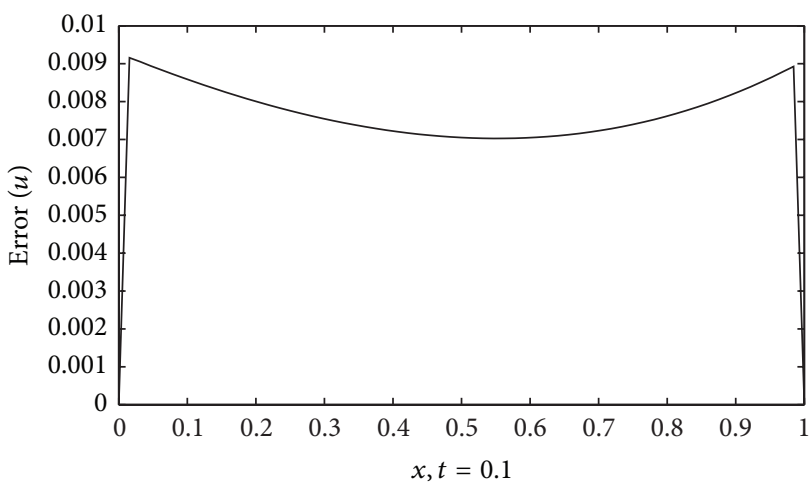

(c) Interval wavelet collocation method with constant $L=1$

FIGURE 3: Numerical errors comparison among the dynamic, static interval wavelet method and the finite difference method $(j=6, \alpha=0.6)$.

TABLE 1: Condition number of the Fokker-Planck equation.

\begin{tabular}{|c|c|c|c|c|c|c|c|}
\hline \multirow{3}{*}{$j$} & \multirow{3}{*}{$\alpha$} & \multicolumn{3}{|c|}{$\tau=0.0001$} & \multicolumn{3}{|c|}{$\tau=0.00001$} \\
\hline & & \multirow{2}{*}{ Interval FDM } & \multicolumn{2}{|c|}{ Interval wavelet } & \multirow{2}{*}{ Interval FDM } & \multicolumn{2}{|c|}{ Interval wavelet } \\
\hline & & & $L=1$ & $L=2$ & & $L=1$ & $L=2$ \\
\hline \multirow{3}{*}{4} & 0.8 & 1.9730 & 2.5810 & 2.9399 & 1.1364 & 1.2461 & 1.2738 \\
\hline & 0.6 & 11.3319 & 11.8703 & 20.8760 & 2.6645 & 3.5347 & 4.3019 \\
\hline & 0.4 & 198.8737 & 91.0582 & 365.5470 & 43.4632 & 31.2993 & 80.5876 \\
\hline \multirow{3}{*}{5} & 0.8 & 6.2479 & 7.8009 & 11.6382 & 1.5798 & 2.0236 & 2.2010 \\
\hline & 0.6 & 83.0421 & 52.1757 & 160.0987 & 10.9216 & 12.0887 & 20.8948 \\
\hline & 0.4 & 1912.4 & 476.7221 & 3632.5 & 380.6050 & 152.3718 & 727.8708 \\
\hline \multirow{3}{*}{6} & 0.8 & 39.1651 & 31.2950 & 76.7074 & 39.1651 & 5.2458 & 6.9255 \\
\hline & 0.6 & 764.3693 & 255.2801 & 1476.4 & 79.4793 & 51.7012 & 155.2663 \\
\hline & 0.4 & 19847.0000 & 2574.7 & 37997 & 3769.1 & 790.3041 & 7238.0 \\
\hline \multirow{3}{*}{7} & 0.8 & 340.7877 & 145.7761 & 663.4654 & 19.8525 & 19.3224 & 38.9722 \\
\hline & 0.6 & 7757.6000 & 1333.9 & 14931 & 730.3466 & 249.7668 & 1416.8 \\
\hline & 0.4 & 214660.0000 & 14202 & 415100 & 39724 & 4266.4 & 76386 \\
\hline
\end{tabular}

to $L=1$ (Figure $1(\mathrm{~b})$ ). FFPE is a 2 -order PDEs with respect to $x$, so $L \geq 2$ is the necessary condition for the interval wavelet satisfying the requirement of FFPE. We also noticed that the condition number of FFPE from the Table 1 that the condition number of $L=2$ increases more rapid than $L=1$ with the increase of $j$ and the decrease of $\alpha$. It has been mentioned in Section 2 that the larger condition number can decrease the calculation precision greatly. This also can be illustrated in Figure 2. The condition number in Figure 2(a) is greatly larger than in Figure 2(b), although the 
TABLE 2: Influence of $\alpha$ on the numerical precision $(t=0.0001, T=0.1)$.

\begin{tabular}{|c|c|c|c|c|c|c|c|}
\hline \multirow{2}{*}{$j$} & \multirow{2}{*}{$\alpha$} & \multicolumn{3}{|c|}{$e_{1}$} & \multicolumn{3}{|c|}{$e_{2}$} \\
\hline & & Interval FDM & $\begin{array}{c}\text { Interval WCM } \\
\quad(L=2)\end{array}$ & $\begin{array}{c}\text { Dynamic } \\
\text { interval WCM }\end{array}$ & Interval FDM & $\begin{array}{l}\text { Interval WCM } \\
\quad(L=2)\end{array}$ & $\begin{array}{c}\text { Dynamic } \\
\text { interval WCM }\end{array}$ \\
\hline \multirow{3}{*}{4} & 0.8 & $5.5367 \times 10^{-6}$ & $8.1588 \times 10^{-5}$ & $5.5920 \times 10^{-6}$ & $4.1037 \times 10^{-6}$ & $5.8298 \times 10^{-5}$ & $4.1514 \times 10^{-6}$ \\
\hline & 0.6 & $5.7907 \times 10^{-6}$ & $4.1158 \times 10^{-4}$ & $7.1813 \times 10^{-6}$ & $4.2424 \times 10^{-6}$ & $2.9468 \times 10^{-4}$ & $5.5971 \times 10^{-6}$ \\
\hline & 0.4 & $3.5309 \times 10^{-6}$ & $9.1673 \times 10^{-4}$ & $3.7967 \times 10^{-5}$ & $2.6232 \times 10^{-6}$ & $6.3692 \times 10^{-4}$ & $2.1348 \times 10^{-5}$ \\
\hline \multirow{3}{*}{5} & 0.8 & $5.5551 \times 10^{-6}$ & $8.2118 \times 10^{-5}$ & $5.8424 \times 10^{-6}$ & $4.1718 \times 10^{-6}$ & $5.9148 \times 10^{-5}$ & $4.4649 \times 10^{-6}$ \\
\hline & 0.6 & $5.7760 \times 10^{-6}$ & $4.0932 \times 10^{-4}$ & $8.9707 \times 10^{-6}$ & $4.2907 \times 10^{-6}$ & $2.9753 \times 10^{-4}$ & $7.5330 \times 10^{-6}$ \\
\hline & 0.4 & $6.5910 \times 10^{264}$ & 0.3971 & 0.0585 & inf & 0.1195 & 0.0491 \\
\hline \multirow{3}{*}{6} & 0.8 & $5.5563 \times 10^{-6}$ & $8.1965 \times 10^{-5}$ & $1.3154 \times 10^{-5}$ & $4.2041 \times 10^{-6}$ & $5.9517 \times 10^{-5}$ & $8.6913 \times 10^{-6}$ \\
\hline & 0.6 & $3.7124 \times 10^{265}$ & 0.0554 & 0.0096 & inf & 0.0105 & 0.0076 \\
\hline & 0.4 & inf & $1.2637 \times 10^{3}$ & 0.0588 & inf & 375.3305 & 0.0499 \\
\hline \multirow{3}{*}{7} & 0.8 & $3.4932 \times 10^{243}$ & 0.0031 & 0.0019 & inf & $3.8313 \times 10^{-4}$ & $1.2937 \times 10^{-4}$ \\
\hline & 0.6 & inf & 216.5596 & 23.7361 & inf & 40.0263 & 9.3964 \\
\hline & 0.4 & $\inf$ & $1.4462 \times 10^{6}$ & 327.6987 & $\inf$ & $4.2662 \times 10^{5}$ & 21.7694 \\
\hline
\end{tabular}

TABLE 3: Dynamic $L$ and the iteration times at the same $L$ value $(j=$ $6, T=0.1$, and $\tau=0.0001$ ).

\begin{tabular}{lccccc}
\hline$L$ & 3 & 1 & 2 & 3 & 2 \\
\hline Iteration times & 11 & 14 & 4 & 3 & 968 \\
\hline
\end{tabular}

approximation of $L=2$ is better than $L=1$. The former has failed to solve FFPE obviously. In fact, this explained the reason why we construct the dynamic interval wavelet.

The numerical errors comparisons among the dynamic, static interval wavelet method and the interval finite difference method are showen in Figure 3. The result also can be illustrated in Table 2.

The robustness of the dynamic interval wavelet collocation method (DIWCM) is the best compared to the interval FDM and the static interval WCM, as it avoids both of the larger condition number and the error of the approximation simultaneity. The varied process of $L$ is showen in Table 3. It shows that the value of $L$ is fixed at $L=2$ after a short time of vibration. This reflects the properties of the FFPE to some extent.

In addition, it also has to be noticed that we can get the higher precision solution with the interval finite difference method (FDM) as the amount of the collocation points decreases (Figure 4). It is well known that increasing the collocation points can impove the approximation although it can increase the condition number in FFPE. In fact, it profits from the smoothness of the solution, which would not work in solving the nonlinear problems.

All above numerical experiments are done with the Shannon-Gabor wavelet. It is well known that the presence of the Gaussian window destroys the orthogonal properties possessed by the Shannon wavelet, effectively worsening the approximation efficiency to a Dirac delta function. Comparing with the Shannon wavelet collocation method (Figure 5), the Shannon-Gabor wavelet numerical method has higher precision and more complicated calculation amount. But it is showen in Figure 5 that dynamic interpolation wavelet construction scheme can be applied to both of the ShannonGabor wavelet and the Shannon wavelet. As a matter of fact, the dynamic scheme is designed for the interpolation wavelet, which has no connection with certain concrete wavelet function.

\section{Conclusions}

In solving the fractional Fokker-Planck equations, there are two factors related to the choice of $L$. The first factor is the condition number, which relates to the parameters $\alpha, j$ and the time step $\tau$. The larger $L$ can decrease the calculation precision greatly. Another factor is the approximation of the function and its derivatives, especially near the boundary. Using the interval wavelet with constant $L$ to solve the fraction Fokker-Planck equations cannot eliminate the boundary effect completely as the condition number is sensitive to the parameter $\alpha$. With regard to the accuracy and time complexity of the solution in comparison with those obtained with other algorithms, the dynamic interval wavelet on $L$ constructed in this paper is more reasonable. The numerical experiments illustrate that it is necessary to construct the dynamic interval wavelet collocation method for the fractional PDEs. 

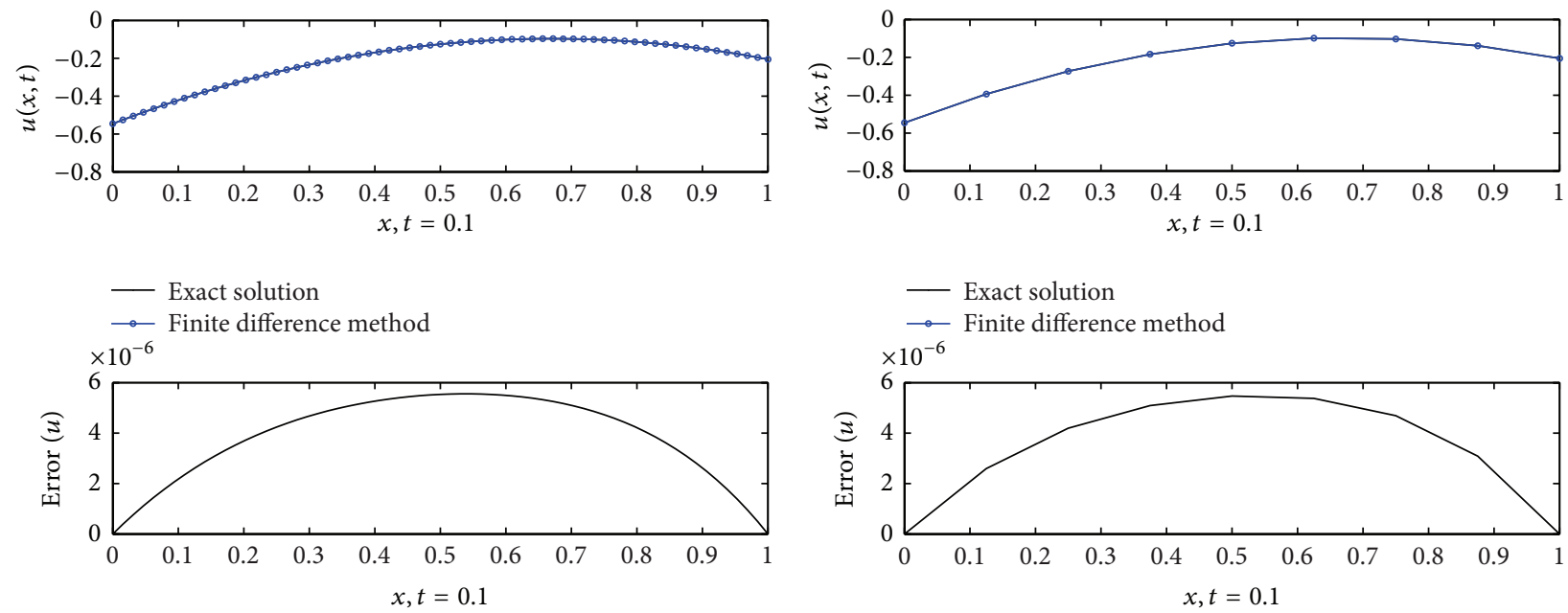

(a) $j=6, e_{1}=5.5563 \times 10^{-6}, e_{2}=4.2041 \times 10^{-6}$, and condition number is 39.1651

(b) $j=3, e_{1}=5.4691 \times 10^{-6}, e_{2}=3.9526 \times 10^{-6}$, and condition number is 1.2202

FIGURE 4: Finite-difference method $(\alpha=0.8)$.
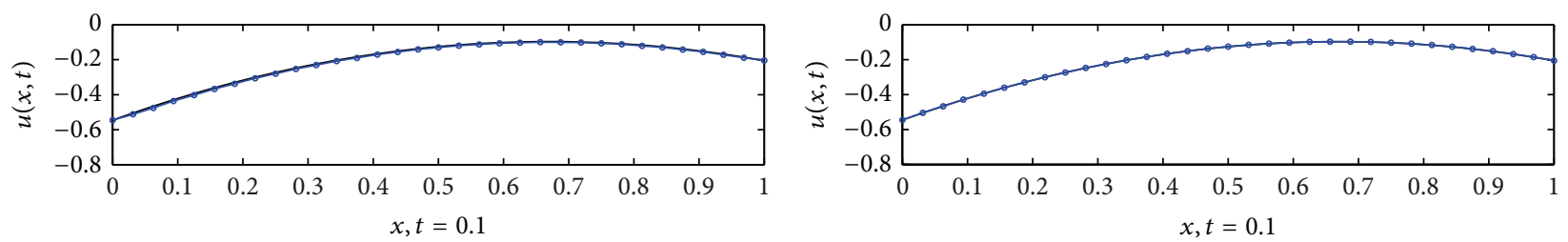

- Exact solution

$\therefore$ Wavelet numerical method

- Exact solution

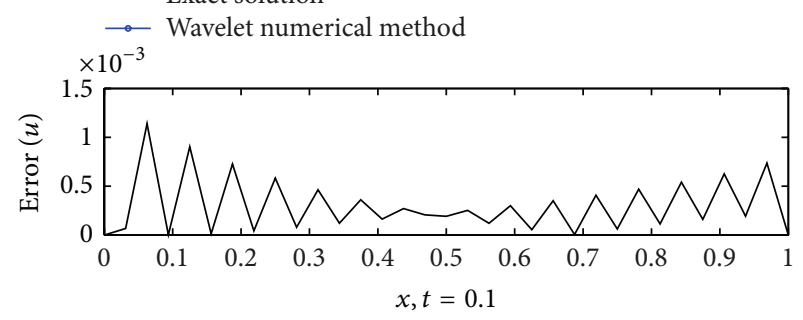

(a) Static interval Shannon wavelet collocation method, $j=5$, and $e_{1}=$ $9.95023 \times 10^{-3}, e_{2}=5.23917 \times 10^{-3}$

(b) Dynamic interval Shannon wavelet collocation method, $j=5, e_{1}=$ $1.1392 \times 10^{-3}$, and $e_{2}=4.0893 \times 10^{-4}$

Figure 5: Comparison between the static and dynamic interval Shannon wavelet collocation method $(\alpha=0.8)$.

\section{Acknowledgments}

This work is supported by the National Natural Science Foundation of China (no. 41171337) and the National High Technology Research and Development Program of China (no. 2006AA10Z235).

\section{References}

[1] M. Li and W. Zhao, "On 1/ $f$ noise," Mathematical Problems in Engineering, vol. 2012, Article ID 673648, 23 pages, 2012.

[2] R. Metzler, E. Barkai, and J. Klafter, "Anomalous diffusion and relaxation close to thermal equilibrium: a fractional fokkerplanck equation approach," Physical Review Letters, vol. 82, no. 18, pp. 3563-3567, 1999.
[3] S. Chen, F. Liu, P. Zhuang, and V. Anh, "Finite difference approximations for the fractional fokker-planck equation," Applied Mathematical Modelling, vol. 33, no. 1, pp. 256-273, 2009.

[4] E. G. Bakhoum and C. Toma, "Modeling transitions in complex systems by multiplicative effect of temporal patterns extracted from signal flows," Mathematical Problems in Engineering, vol. 2012, Article ID 409856, 11 pages, 2012.

[5] C. Toma, "Advanced signal processing and command synthesis for memory-limited complex systems," Mathematical Problems in Engineering, vol. 2012, Article ID 927821, 13 pages, 2012.

[6] E. G. Bakhoum and C. Toma, "Transient aspects of wave propagation connected with spatial coherence," Mathematical Problems in Engineering, vol. 2013, Article ID 691257, 5 pages, 2013. 
[7] S. X. Hu, Z. W. Liao, and W. F. Chen, "Sinogram restoration for low-dosed $\mathrm{x}$-ray computed tomography using fractional-order perona-malik diffusion," Mathematical Problems in Engineering, vol. 2012, Article ID 391050, 13 pages, 2012.

[8] J. H. He, "Asymptotic methods for solitary solutions and compactons," Abstract and Applied Analysis, vol. 2012, Article ID 916793, 130 pages, 2012.

[9] M. Li, "Fractal time series-a tutorial review," Mathematical Problems in Engineering, vol. 2010, Article ID 157264, 26 pages, 2010.

[10] M. Li, S. C. Lim, and S. Y. Chen, "Exact solution of impulse response to a class of fractional oscillators and its stability," Mathematical Problems in Engineering, vol. 2011, Article ID 657839, 9 pages, 2011.

[11] M. Li, "Approximating ideal filters by systems of fractional order," Computational and Mathematical Methods in Medicine, vol. 2012, Article ID 365054, 6 pages, 2012.

[12] M. Li and W. Zhao, "Solving Abel's type integral equation with Mikusinski's operator of fractional order," Advances in Mathematical Physics, vol. 2013, Article ID 806984, 4 pages, 2013.

[13] M. Li, C. Cattani, and S. Y. Chen, "Viewing sea level by a onedimensional random function with long memory," Mathematical Problems in Engineering, vol. 2011, Article ID 654284, 13 pages, 2011.

[14] M. Li and W. Zhao, "Visiting power laws in cyber-physical networking systems," Mathematical Problems in Engineering, vol. 2012, Article ID 302786, 13 pages, 2012.

[15] I. Daubechies and G. Teschke, "Variational image restoration by means of wavelets: simultaneous decomposition, deblurring, and denoising," Applied and Computational Harmonic Analysis, vol. 19, no. 1, pp. 1-16, 2005.

[16] S. L. Mei, "Construction of target controllable image segmentation model based on homotopy perturbation technology," Abstract and Applied Analysis, vol. 2013, Article ID 131207, 8 pages, 2013.

[17] S.-L. Mei, "HPM-based dynamic wavelet transform and its application in image denoising," Mathematical Problem in Engineering, vol. 2013, Article ID 309418, 10 pages, 2013.

[18] H. Yan, "Adaptive wavelet precise integration method for nonlinear black-scholes model based on variational iteration method," Abstract and Applied Analysis, vol. 2013, Article ID 735919, 6 pages, 2013.

[19] L. W. Liu, "Interval wavelet numerical method on fokkerplanck equations for nonlinear random system," Advances in Mathematical Physics, vol. 2013, Article ID 651357, 7 pages, 2013.

[20] C. Cattani and A. Ciancio, "Separable transition density in the hybrid model for tumor-immune system competition," Computational and Mathematical Methods in Medicine, vol. 2012, Article ID 610124, 6 pages, 2012.

[21] C. Cattani, A. Ciancio, and B. Lods, "On a mathematical model of immune competition," Applied Mathematics Letters, vol. 19, no. 7, pp. 678-683, 2006.

[22] C. Cattani, "Fractional calculus and shannon wavelet," Mathematical Problems in Engineering, vol. 2012, Article ID 502812, 26 pages, 2012.

[23] C. Cattani, "Shannon wavelets for the solution of integrodifferential equations," Mathematical Problems in Engineering, vol. 2010, Article ID 408418, 22 pages, 2010.

[24] R. Y. Xing, "Wavelet-based homotopy analysis method for nonlinear matrix system and its application in Burgers equation,"
Mathematical Problems in Engineering, vol. 2013, Article ID 982810, 7 pages, 2013.

[25] S.-L. Pang, "Wavelet numerical method for nonlinear random system," Transactions of the Chinese Society of Agricultural Machinery, vol. 38, no. 3, pp. 168-170, 2007.

[26] S. M. Bibic, "Harmonic wavelet analysis-connection coefficients for nonlinear PDE," UPB Scientific Bulletin, Series A, vol. 73, no. 1, pp. 27-36, 2011.

[27] M. Bieri, "A sparse composite collocation finite element method for elliptic SPDEs," SIAM Journal on Numerical Analysis, vol. 49, no. 6, pp. 2277-2301, 2011.

[28] N. A. Libre, A. Emdadi, E. J. Kansa, M. Shekarchi, and M. Rahimian, "A fast adaptive wavelet scheme in RBF collocation for nearly singular potential PDEs," Computer Modeling in Engineering and Sciences, vol. 38, no. 3, pp. 263-284, 2008.

[29] C. Cattani, "Second order shannon wavelet approximation of $C^{2}$-functions," UPB Scientific Bulletin, Series A, vol. 73, no. 3, pp. 73-84, 2011.

[30] C. Cattani and L. M. S. Ruiz, "Discrete differential operators in multidimensional haar wavelet spaces," International Journal of Mathematics and Mathematical Sciences, vol. 2004, no. 44, pp. 2347-2355, 2004.

[31] D. K. Hoffman, G. W. Wei, D. S. Zhang, and D. J. Kouri, "Shannon-Gabor wavelet distributed approximating functional," Chemical Physics Letters, vol. 287, no. 1-2, pp. 119-124, 1998.

[32] O. V. Vasilyev and S. Paolucci, "A dynamically adaptive multilevel wavelet collocation method for solving partial differential equations in a finite domain," Journal of Computational Physics, vol. 125, no. 2, pp. 498-512, 1996.

[33] S. L. Mei, H. L. Lv, and Q. Ma, "Construction of interval wavelet based on restricted variational principle and its application for solving differential equations," Mathematical Problems in Engineering, vol. 2008, Article ID 629253, 14 pages, 2008.

[34] S. L. Mei, C. J. Du, and S. W. Zhang, "Asymptotic numerical method for multi-degree-of-freedom nonlinear dynamic systems," Chaos, Solitons and Fractals, vol. 35, no. 3, pp. 536-542, 2008.

[35] S. L. Mei, Q. S. Lu, S. W. Zhang, and L. Jin, "Adaptive interval wavelet precise integration method for partial differential equations," Applied Mathematics and Mechanics, vol. 26, no. 3, pp. 364-371, 2005.

[36] S. L. Mei, C. J. Du, and S. W. Zhang, "Linearized perturbation method for stochastic analysis of a rill erosion model," Applied Mathematics and Computation, vol. 200, no. 1, pp. 289-296, 2008.

[37] S.-L. Mei, "HAM-based adaptive multi-scale meshless method for Burgers equation," Journal of Applied Mathematics, vol. 2013, Article ID 248246, 10 pages.

[38] T. D. Frank, "Autocorrelation functions of nonlinear fokkerplanck equations," European Physical Journal B, vol. 37, no. 2, pp. 139-142, 2004. 


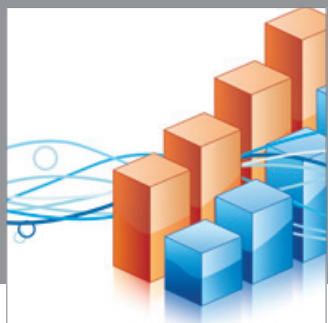

Advances in

Operations Research

mansans

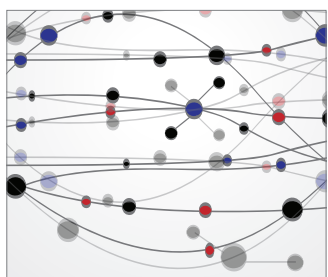

The Scientific World Journal
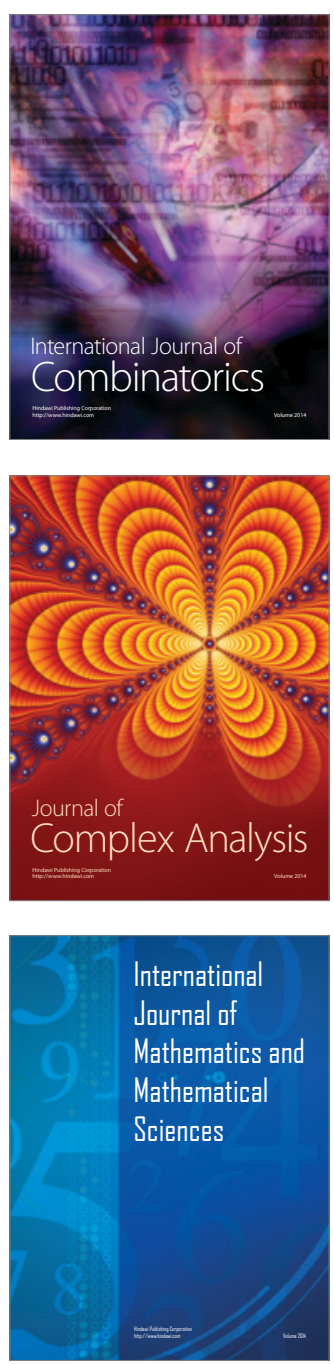
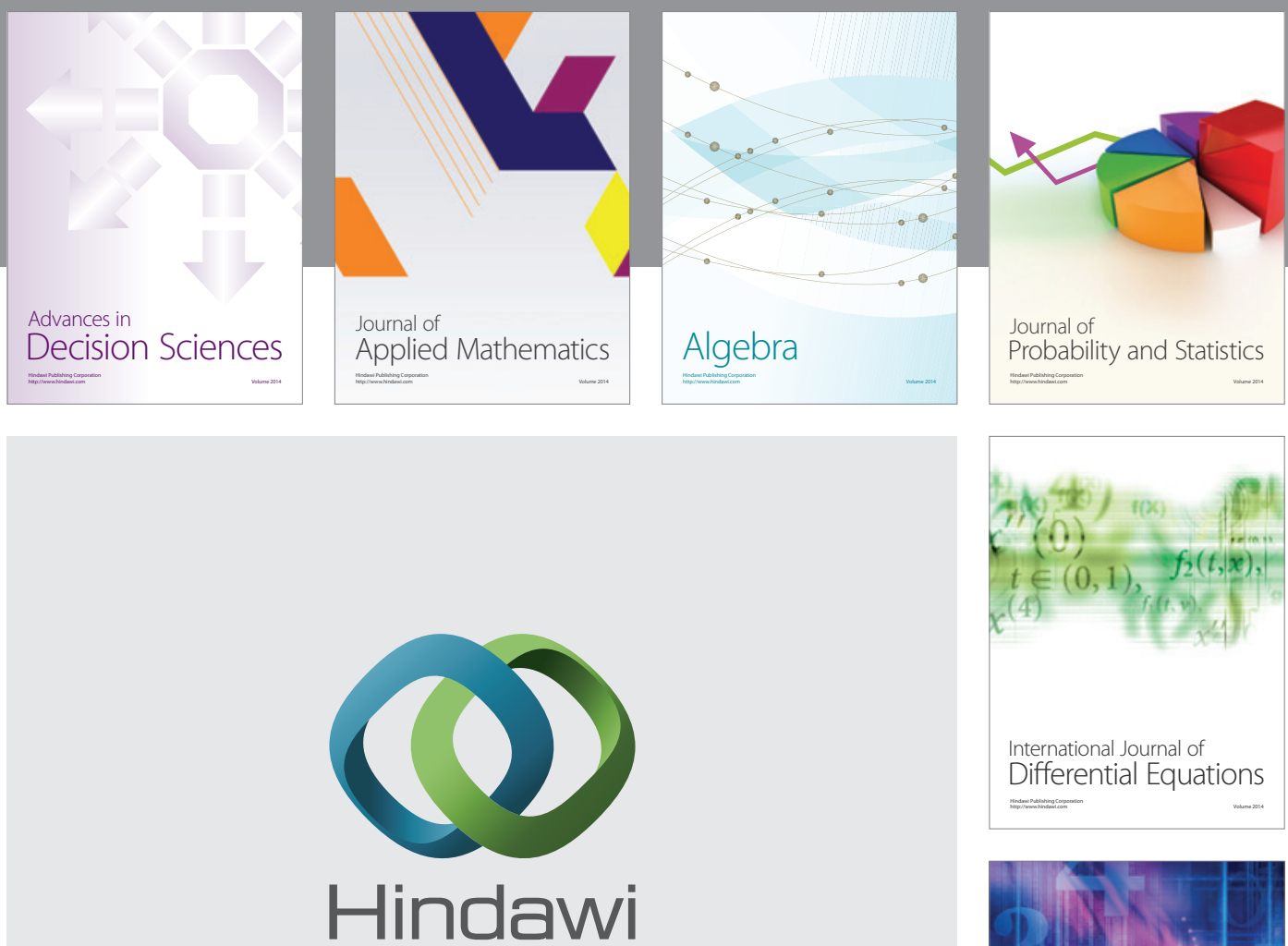

Submit your manuscripts at http://www.hindawi.com
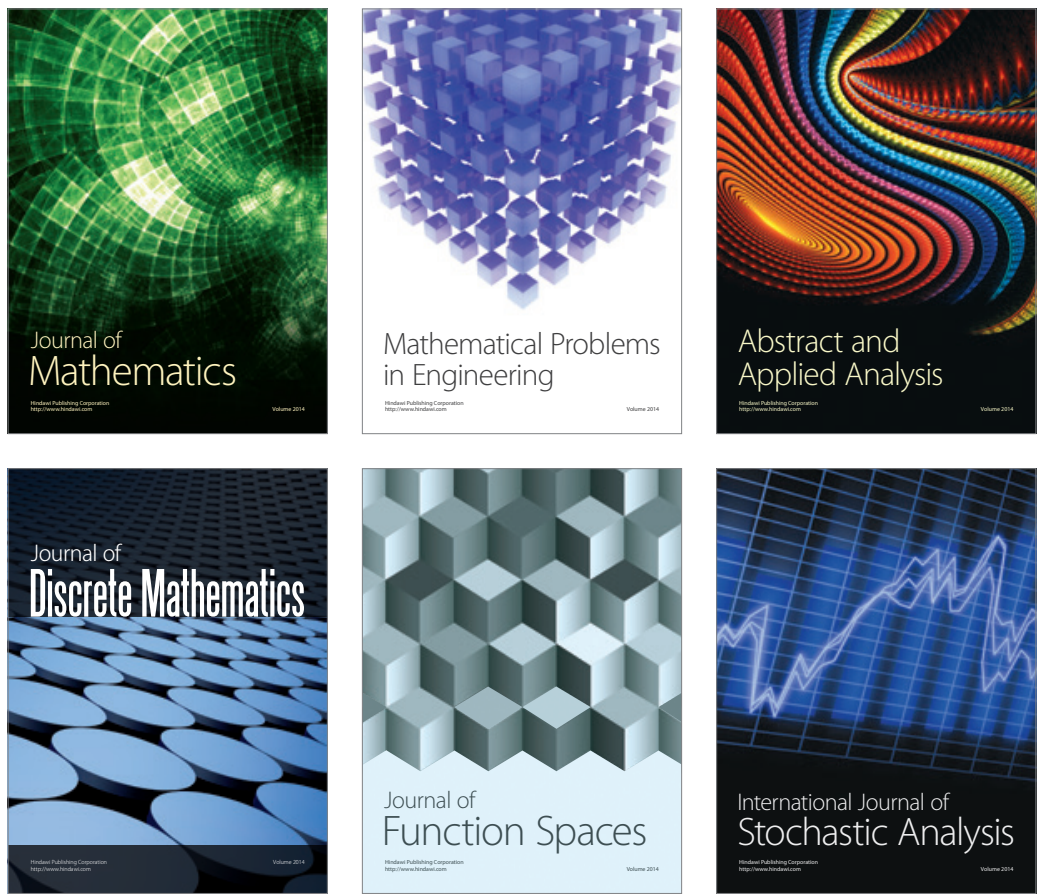

Journal of

Function Spaces

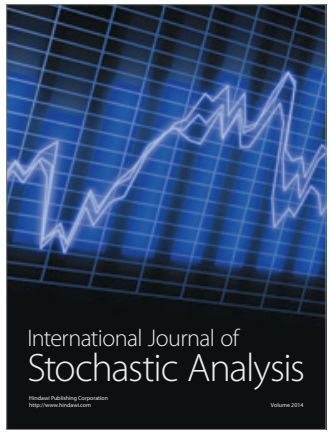

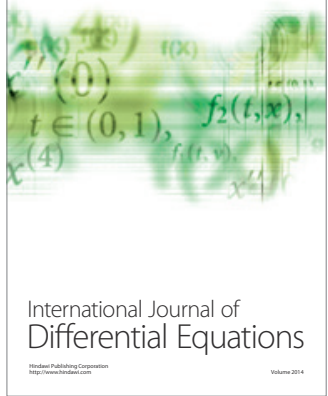
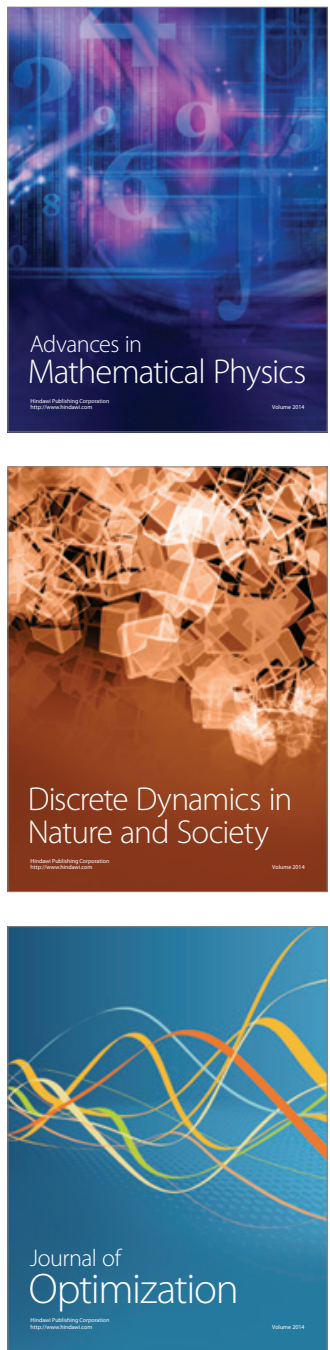